\title{
Du lexique de l'eau dans Mont-Oriol et Pierre et Jean de Guy de Maupassant
}

\section{Benzina, Ouafae}

Institut d'Etudes et de Recherches pour l’Arabisation, Université Mohammed V-Rabat, wafae ben2000@yahoo.fr

\begin{abstract}
Resumen
Nuestro trabajo se articula alrededor del tema del agua en Mont-Oriol y Pierre y Jean de Guy de Maupassant. En efecto, estas dos obras novelescas rebosan agua en todas sus formas. En Mont-Oriol, el elemento acuático sólo le determina su espacio novelesco y constituye el marco de la novela. Está presente bajo varios aspectos : agua, fuente, lago, estación, arroyo. En Pierre y Jean, el léxico del mar es muy abundante. El tema del mar vuelve sin cesar en la novela. Aparece en inicio y final, evocando largamente los barcos y la pesca. Las imágenes del agua en estas dos obras novelescas reflejan el amor del autor normando para este elemento natural. Con el fin de analizar este brote del léxico del agua en estas dos obras, explotaremos la función Contexto y la del vocabulario específico del software Hyperbase, el software de explotación estadística documental y de tratamiento cuantitativo de grandes corpus, elaborado por Étienne Brunet. En efecto, nuestro enfoque para el tratamiento de nuestro corpus por este software había acabado en una base de datos que llamamos Maupassa.exe. En la presentación del vocabulario específico de cada obra, se trata de señalar las unidades cuya frecuencia manifiesta un excedente o un déficit significativo. Para determinar el vocabulario específico de un corpus, el software Hyperbase calcula la desviación (diferencia o variación) reducida de cada forma en cada una de las partes del corpus. El botón Contexto permite ver el caso que se busca en el contexto del párrafo. Si se hace clic en uno de los pasos fijados, tenemos la posibilidad de ver la página entera donde la palabra aparece. Vamos pues a basarnos en esta función que hace ganar a los investigadores un tiempo considerable con el fin de determinar el sentido que Maupassant da al agua en todas sus formas.
\end{abstract}

Palabras clave : léxico ; agua ; Mont-Oriol ; Pierre y Jean ; Maupassant.

\section{Résumé}

Notre travail s'articule autour du thème de l'eau dans Mont-Oriol et Pierre et Jean de Guy de Maupassant. En effet, ces deux cuvres romanesques regorgent d'eau dans toutes ses formes. Dans Mont-Oriol, l'élément aquatique détermine son espace romanesque et constitue à lui seul le cadre du roman. Il est présent sous plusieurs aspects : eau, source, lac, station, ruisseau. Dans Pierre et Jean, le lexique de la mer est fort abondant. Le thème de la mer revient sans cesse dans le roman. Il y apparaît en ouverture et en conclusion, évoquant longuement les bateaux et la pêche. Les images de l'eau dans ces deux œuvres romanesques reflètent l'amour de l'auteur normand pour cet élément naturel. Afin d'analyser ce jaillissement du lexique de l'eau dans ces deux œuvres, nous exploiterons la fonction Contexte et celle du vocabulaire spécifique du logiciel Hyperbase, un logiciel d'exploitation statistique documentaire et de traitement quantitatif des grands corpus, élaboré par Étienne Brunet. En effet, notre démarche pour le traitement de notre corpus par ce logiciel avait abouti à une base de données que nous avons appelée Maupassa.exe. Dans la présentation du vocabulaire spécifique de chaque æuvre, il s'agit de signaler les unités dont la fréquence manifeste un excédent ou un déficit significatif. Pour déterminer le vocabulaire spécifique d'un corpus, le logiciel Hyperbase calcule l'écart réduit de chaque forme dans chacune des parties du corpus. La mise en œuvre du bouton Contexte permet de voir l'occurrence que l'on cherche dans le contexte du paragraphe. Si l'on clique sur l'un des passages affichés, on a la possibilité de voir la page entière où le mot apparaît. Nous allons donc nous baser sur cette fonction en vue de déterminer le sens que Maupassant donne à l'eau dans toutes ses formes.

Mots-clés : lexique ; eau ; Pierre et Jean ; Mont-Oriol ; Maupassant. 


\begin{abstract}
The following article revolves around the theme of water in Mont-Oriol and Pierre and Jean of Guy de Maupassant. These two fabulous novels, indeed, include the theme of water in all its forms. In Mont-Oriol, the aquatic element determines its novelistic space and represents in itself the novel's frame. It is present in many aspects : water, source, lake, resort, and stream. In Pierre and Jean, the lexicon of the sea is very abundant. The theme of the sea keeps appearing continuously in the novel. It appears during the opening and the closure, evoking for a long time boats and fishing. In these two novels, images of water reflect the love of the Norman author for this natural element. To analyze this gush of the lexicon of water in these two works, we will operate the function Contexte and the specific vocabulary of Hyperbase software, which is documentary statistical operating software and quantitative processing of large corpora, developed by Étienne Brunet. Indeed, our approach to the treatment of our corpus by this program had resulted in a database which we called Maupassa.exe. The presentation of the specific vocabulary of each work is reporting units whose frequency shows a surplus or a significant deficit. The Hyperbase software calculates the reduced gap of every form in every part of the corpus, in order to determine the specific vocabulary of a corpus. The implementation of the Contexte button allows seeing the occurrence that one seeks in the context of the paragraph. If we click on one of the displayed passages, one has the opportunity to see the whole page where the word appears. Based on this function, we will determine the meaning that Maupassant gives to water in all its forms.
\end{abstract}

Keywords : lexicon ; water, Mont-Oriol ; Pierre and Jean ; Maupassant.

« La langue française, d'ailleurs, est une eau pure que les écrivains maniérés n’ont jamais pu et ne pourront jamais troubler. » (Maupassant, Le Roman (préface de Pierre et Jean), 1999 : 32).

Considérée comme source de la vie, l'eau symbolise la pureté et la fertilité, la fraicheur et l'humidité. L'eau est à la fois calme et violente. Elle soutient et détruit. Les représentations de l'eau se manifestent dans toutes formes d'art. On la trouve entre autres dans la peinture, la sculpture, le cinéma, la poésie et dans le roman. L'élément aquatique a inspiré des poètes et des romanciers en le représentant sous plusieurs formes. Des représentations qui déterminent l’humeur générale de l'image.

Parmi ces écrivains dont la vie, les nouvelles et les romans ont été marqués par l'eau, il y a Guy de Maupassant. En effet, l'eau a joué un rôle primordial dans la vie de l'auteur normand. Amoureux de cet élément naturel, Maupassant prend du plaisir à parler de l'eau dans ses œuvres. Elle représente un élément fondamental pour lui. L'eau est omniprésente dans son œuvre dès ses débuts littéraires. Ses premiers vers publiés s'appellent : Au bord de l'eau. Une de ses nouvelles connues s'intitule : Sur l'eau. C'est dans cette nouvelle que Maupassant exprime son amour pour l'eau : « J'aime le ciel comme un oiseau, les forêts comme un loup rôdeur, les rochers comme un chamois, l'herbe profonde pour m'y rouler, pour y courir comme un cheval, et l'eau limpide pour y nager comme un poisson. » (2006: 123).

Aussi, dans les romans de Maupassant l'eau se manifeste-t-elle sous plusieurs aspects : mer, pluie, larmes, neige, etc. Elle est fort présente dans Une vie, Pierre et Jean et Mont-Oriol. En effet, ces œuvres romanesques regorgent d'eau dans toutes ses formes. Dans Pierre et Jean, le lexique de la mer est fort abondant. Le thème de la mer revient sans cesse dans le roman. Il y apparaît en ouverture et en conclusion. Dans Mont-Oriol, l'élément aquatique détermine son espace romanesque et constitue à lui seul le cadre du roman. Cette dominance de l'eau génère donc toute l'action. Elle est présente sous plusieurs aspects : eau, source, lac, station, ruisseau, etc.

Afin d'analyser ce jaillissement du lexique de l'eau dans Pierre et Jean et Mont-Oriol, nous exploiterons la fonction Contexte et celle du vocabulaire spécifique du logiciel Hyperbase, un logiciel d'exploitation statistique documentaire et de traitement quantitatif des grands corpus, élaboré par Étienne Brunet. Le traitement de notre corpus par ce logiciel avait abouti à une base de données que nous avons appelée Maupassa.exe. Dans cette base, nous avons regroupé toute l'œuvre romanesque de Guy de Maupassant : Une vie, Bel-Ami, Mont-Oriol, Pierre et Jean, Fort comme la mort et Notre cœur. 
Le logiciel Hyperbase offre la possibilité d'étudier le corpus dans son intégralité ou se limiter au(x) texte(s) sélectionné(s). Pour cette contribution, nous allons nous focaliser surtout sur Mont-Oriol et Pierre et Jean.

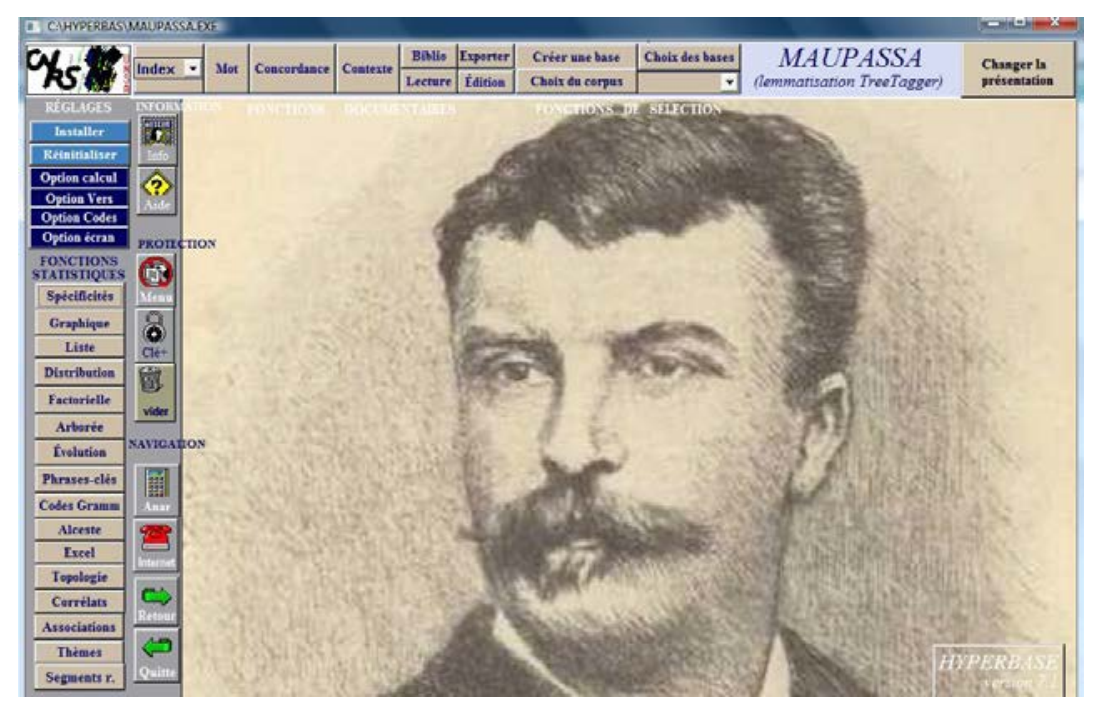

Fig. 1 Base Maupassa.exe

\section{Vocabulaire spécifique de Mont-Oriol}

Il s’agit de signaler les unités dont la fréquence manifeste un excédent ou un déficit significatif à travers une comparaison avec les autres textes du corpus. En établissant les listes des mots spécifiques de chaque texte, nous pourrons relever leurs thèmes. Pour déterminer le vocabulaire spécifique d’un corpus, le logiciel Hyperbase calcule l'écart réduit de chaque forme dans chacune des parties du corpus : «Chacun des textes est considéré isolément, dans son individualité propre. L’écart réduit nous donne là encore le moyen approprié pour distinguer ce qui est significatif. » (Brunet, 1988 : 441).

Ainsi, le calcul des spécificités permet de repérer plus facilement la thématique dominante. Comme l'écrit Étienne Brunet : «Le calcul des spécificités sert également à extraire les passages caractéristiques d’un texte du corpus. Le principe est simple : seront retenus les extraits qui contiennent le plus grand nombre de termes spécifiques. » (2009 : 54 ).

Les listes ci-dessous présentent le vocabulaire excédentaire ou déficitaire dans Mont-Oriol. Les trois colonnes de chiffres qui y figurent représentent respectivement le nombre d'occurrences de chaque forme dans le texte, leur total dans le corpus et la valeur de l'écart réduit par rapport au corpus total.

Tableau 1. Vocabulaire spécifique Mont-Oriol (formes)

\begin{tabular}{lccl}
\hline Écart & Corpus & Texte & Mot \\
\hline 31.0 & 224 & 222 & Gontran \\
12.2 & 42 & 42 & Enval \\
12.2 & 155 & 95 & Marquis \\
11.3 & 37 & 37 & Bonnefille \\
11.2 & 36 & 36 & Casino \\
11.1 & 65 & 52 & Louise \\
11.1 & 51 & 45 & établissement \\
11.1 & 46 & 42 & Eaux \\
10.2 & 63 & 48 & Sœur
\end{tabular}


Du lexique de l'eau dans Mont-Oriol et Pierre et Jean de Guy de Maupassant

\begin{tabular}{llll}
9.9 & 54 & 43 & Source \\
8.9 & 114 & 63 & médecin \\
8.7 & 37 & 31 & malades \\
8.7 & 35 & 30 & Bains \\
8.5 & 70 & 45 & Mont \\
7.6 & 21 & 20 & baigneurs \\
7.3 & 17 & 17 & Butte \\
7.2 & 24 & 21 & Colosse \\
7.1 & 33 & 25 & médecins \\
7.0 & 25 & 21 & Aînée \\
7.0 & 23 & 20 & Sources \\
6.5 & 39 & 26 & Montagne \\
6.1 & 265 & 91 & Eau \\
6.1 & 105 & 47 & Hôtel \\
5.8 & 42 & 25 & Bain \\
5.8 & 22 & 17 & Station \\
5.6 & 23 & 17 & Lac \\
4.6 & 51 & 24 & Vin \\
4.4 & 23 & 14 & ruisseau \\
\hline & & &
\end{tabular}

Dans cette liste du vocabulaire spécifique de Mont-Oriol, le registre de l'eau est prédominant : eau, source, bain, baigneur, lac, station, ruisseau.

La distribution du terme eau par rapport aux autres œuvres romanesques de Guy de Maupassant montre l'abondance de l'élément aquatique dans Mont-Oriol.

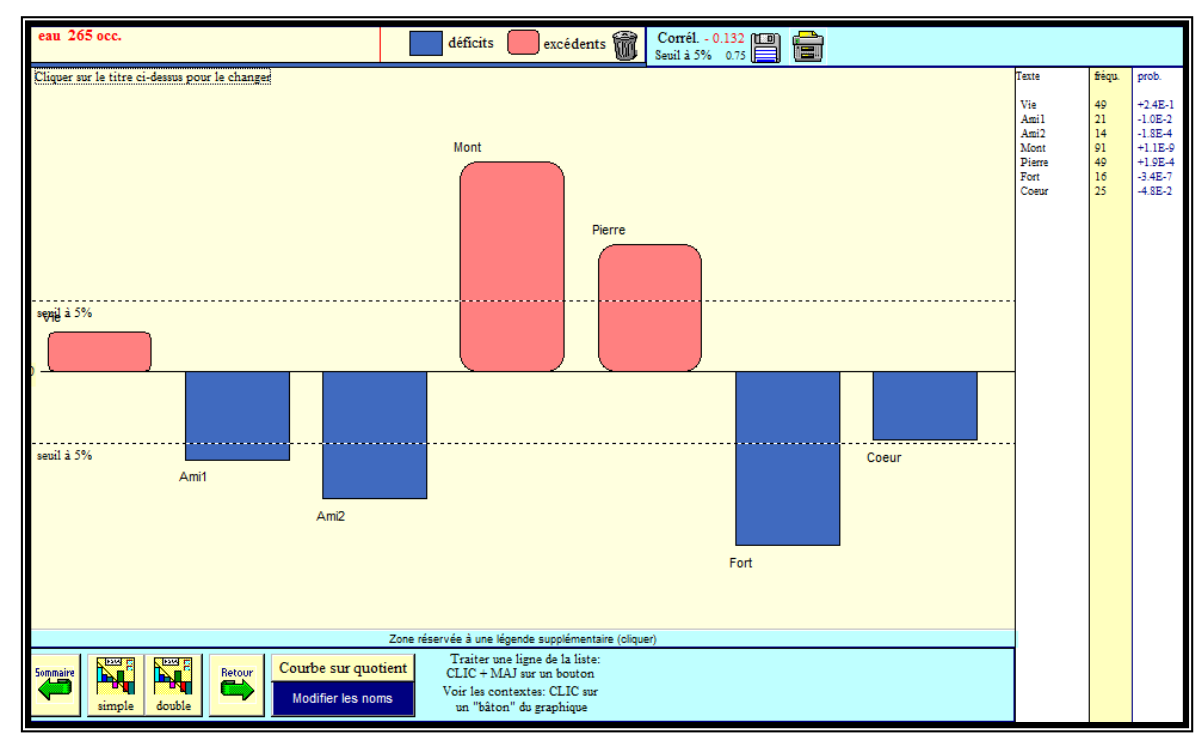

Fig. 2 Distribution du terme eau 
Le terme eau est donc majoritaire dans le quatrième roman de Maupassant avec 91 occurrences. Il résulte que Mont-Oriol est un roman aquatique. En effet, cette œuvre se distingue par son lieu d'action. C’est le seul roman qui ne se déroule ni en Normandie ni à Paris. Son paysage est unique. Il se situe dans une station imaginaire, d'eaux thermales, appelée MontOriol.

Dans une petite station thermale d'Auvergne, Christiane et son mari William Andermatt sont en cure pour remédier à une infécondité. Le père Oriol, un riche paysan, et son fils font exploser une morne sur leur terre. Cette explosion révèle l'existence d'une source. Andermatt se lance dans une série de transactions et parvient à acheter les terrains d'Oriol où se trouvent les sources. Il crée une société, y fait entrer Gontran, le frère de Christiane, et Paul, son ami, comme actionnaires. L'année suivante, Andermatt vient avec Christiane enceinte de Paul contempler son œuvre: Mont-Oriol, fut, ainsi, baptisée la nouvelle station.

Il s'agit donc dans cette œuvre de la découverte d'une source, qui fera du village le lieu de la réussite économique et financière d'Andermatt : « du point de vue de l'organisation spatiale, ce roman est l'histoire d'une rivalité entre deux espaces représentés par les deux stations thermales d’Enval et de Mont-Oriol » (Giacchetti, 1993 : 189).

\section{Images de l'eau dans Mont-Oriol}

Pour exploiter profondément le corpus, le logiciel Hyperbase offre une exploitation méthodique de la documentation. Parmi ces outils, nous citons la fonction Contexte. La mise en œuvre du bouton Contexte, permet de voir l'occurrence que l'on cherche dans le contexte du paragraphe. Si l'on clique sur l'un des passages affichés, on a la possibilité de voir la page entière où le mot apparaît.

L'exploitation de la fonction Contexte du logiciel Hyperbase nous permettra de déterminer les images que Maupassant donne à l'eau.

Les contextes du mot eau figurent ci-dessous :

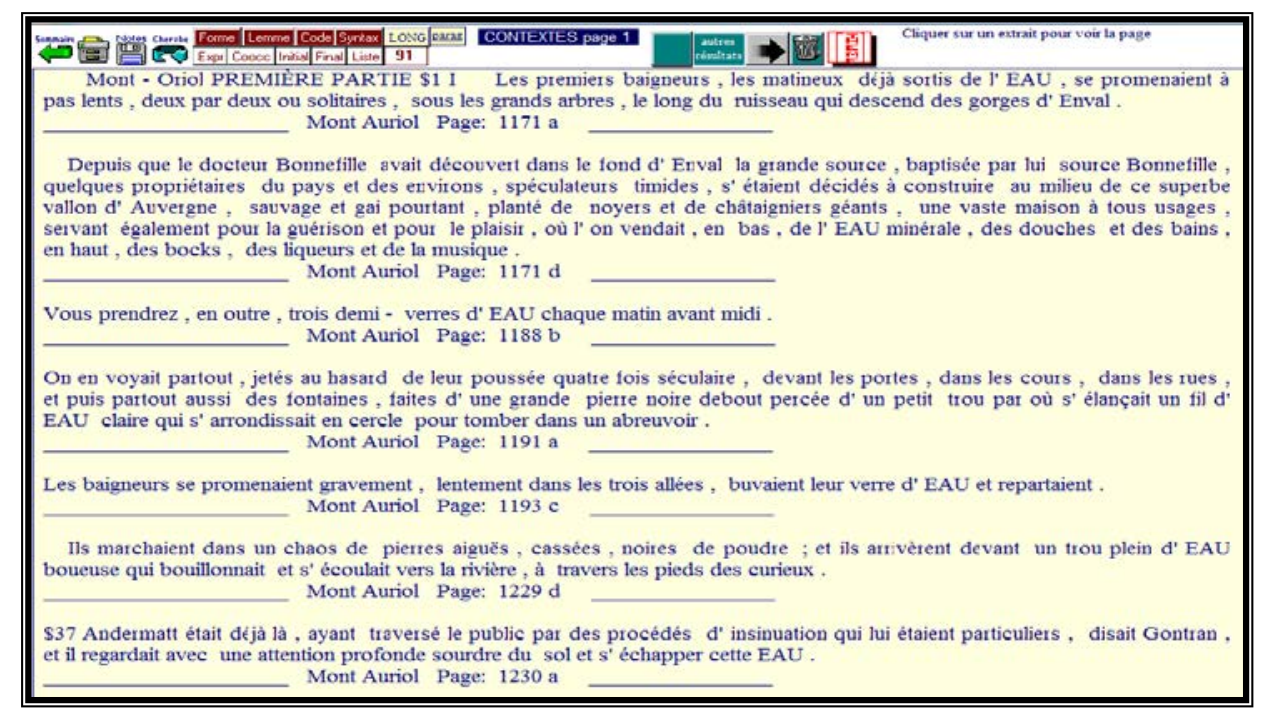

Fig. 3 Contextes du mot eau

Dans ce troisième roman, l'eau est citée dès la première page. Elle est même le fil conducteur de toute l'œuvre. Les eaux de la station sont de l'or, comme l'indique le nom Oriol. Dans Mont-Oriol, l'eau se transforme en argent. Andermatt voit en elle une source de richesse. Il crée alors sa société en achetant les terrains où se trouvent les sources découvertes : «...cette source qui pouvait donner un jour un flot d’argent liquide » (2002: 1267 c).

L'eau jaillit à Mont-Oriol ; prêtres et médecins la boivent et la recommandent aux malades. Les sources de Bonnefille, les sources Louise, Charlotte et Christiane sont miraculeuses : 
L'eau, dans ce roman, n'est pas celle des profondeurs marines, comme dans les deux romans de la dépossession. Contrairement à l'espace marin, l'eau minérale de l'Auvergne est l'eau vive, affectée d'un symbolisme positif : l'eau de la source est, comme l'a bien vu Mary Donaldson-Evans, associée à la fois à la virginité et à la maternité, mais une maternité « bénéfique », celle qui est donneuse d’énergie et non celle qui contient, qui enferme, comme la mer avaleuse (Giachetti, 1993 : 143).

Étant un élément naturel, l'eau est utilisée pour se soigner des maladies chroniques en rétablissant un équilibre naturel dans le corps : «Vous prendrez, en outre, trois demi-verres d'eau chaque matin avant midi » (2002: 1210 c).

Durant sa maladie, Maupassant a essayé les stations thermales pour guérir. Mais l'eau ne l'a pas guéri. Alors pour dénoncer les financiers et les médecins avides qui exploitent les malades crédules, Maupassant critique dans Mont-Oriol le nouveau capitalisme des années 1880 et condamne l'utilisation d'un élément qu'il a toujours chérit et qui lui procure la joie et la sérénité : «Il en est des eaux minérales comme de tout. Il faut qu'on parle d’elles, beaucoup, toujours, pour que les malades en boivent. » (2002: 1443 c).

En effet, les propos sur les affaires sont énormément évoqués dans le roman, ce qui révèle un mal social. La station thermale, censée guérir les maladies, participe à cette maladie avec l'aide des médecins qui jouent la comédie, ne croyant pas eux-mêmes aux bienfaits de ces eaux miraculeuses.

Loin de la satire des stations thermales, l'amoureux de l'eau décrit, par le biais de Christiane, les plaisirs du bain. L'eau offre à l'héroïne de la tranquillité et une sérénité extrême : «Et Christiane se sentait si bien là-dedans, si doucement, si mollement, si délicieusement caressée, étreinte par l'onde agitée, l'onde vivante, l'onde animée de la source qui jaillissait au fond du bassin » (2002 : 1284 c). Pour Christiane, l'eau est symbole de bien-être, de jouissance et de plaisir. Elle (l'eau) semble bien vivante par les bulles de gaz qu'elle contient :

Puis elle enfonça dans l' eau tiède une jambe d'abord, l'autre ensuite, et s'assit dans cette chaleur, dans cette douceur, dans ce bain transparent, dans cette source qui coulait sur elle, autour d'elle, couvrant son corps de petites bulles de gaz, tout le long des jambes, tout le long des bras, et sur les seins aussi. (2002: $1305 \mathrm{~b}$ ).

Ce même plaisir est évoqué dans l'extrait suivant : « Le bain du matin était son premier plaisir, un délicieux plaisir à fleur de peau, une demi-heure exquise dans l'eau chaude et courante qui la disposait à être heureuse jusqu' au soir. » (2002 : 1340 c). L'eau est ainsi pur reflet des sensations de l'héroïne.

Dans Mont-Oriol, l'eau de la source est fécondante. Elle a permis le rapprochement amoureux de Paul et Christiane, venue pour guérir sa stérilité, et elle est fécondante également pour Andermatt en lui permettant un grand succès financier, malgré son impuissance sexuelle.

\section{Vocabulaire spécifique de Pierre et Jean}

Le calcul du vocabulaire spécifique par Hyperbase nous permet d'obtenir le tableau suivant :

Tableau 2 : Vocabulaire spécifique Pierre et Jean (formes)

\begin{tabular}{ccclc}
\hline Écart & Corpus & Texte & Mot \\
\hline 32.0 & 173 & 173 & Roland \\
9.5 & 23 & 22 & Pêche \\
9.3 & 28 & 24 & Port
\end{tabular}




\begin{tabular}{lccl}
8.5 & 30 & 23 & Capitaine \\
7.7 & 462 & 108 & Père \\
7.6 & 52 & Mer \\
7.4 & 155 & 17 & Perle \\
6.7 & 21 & 20 & Bateau \\
6.6 & 34 & 37 & Maman \\
6.5 & 105 & 18 & Roman \\
5.6 & 29 & 16 & Jetée \\
5.0 & 30 & 13 & Navire \\
4.6 & 25 & 11 & Bonhomme \\
4.4 & 21 & 10 & Marée \\
4.3 & 19 & 9 & Navires \\
\hline
\end{tabular}

Le lexique de la mer est fort abondant dans cette liste : mer, pêche, port, capitaine, bateau, jetée, navires, marée. Le tableau suivant présente la distribution du terme mer dans l’œuvre romanesque de Guy de Maupassant.

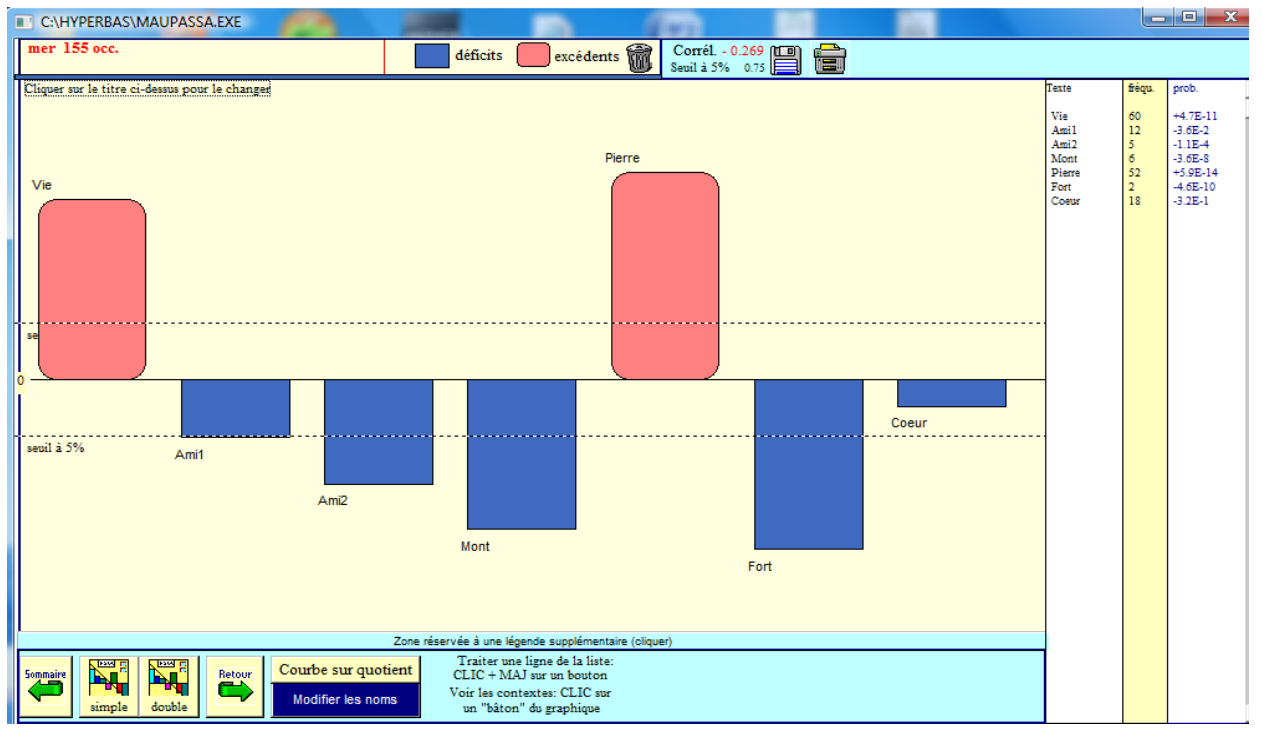

Fig.4 distribution du terme «mer»

Il est donc remarquable que le terme mer a la plus haute fréquence dans Pierre et Jean. Ce qui confirme la dominance de l'élément aquatique dans cette œuvre.

\section{Images de la mer dans Pierre et Jean}

L'eau sous forme de mer a souvent capté l'imaginaire de Maupassant. En effet, depuis son enfance, sa plus grande préoccupation était la mer. Dans son ouvrage, La vie et l'œuvre de Guy de Maupassant, Édouard Maynial témoigne de cet amour de Maupassant pour la mer : 
Je sens, avouait Maupassant, que j’ai dans les veines le sang des écumeurs de mer. Je n’ai pas de joie meilleure par des matins de printemps, que d'entrer avec mon bateau dans des ports inconnus, de marcher tout un jour dans un décor nouveau, parmi des hommes que je côtoie, que je ne reverrai point, que je quitterai le soir venu, pour reprendre la mer, pour m’en aller dormir au 1arge, pour donner le coup de barre du côté de ma fantaisie. (Maynial, 1935 : 37).

Dans Pierre et Jean, le thème de la mer revient sans cesse. Il y apparaît en ouverture et en conclusion, évoquant longuement les bateaux et la pêche. Il représente le fil conducteur de tout le récit. Cette œuvre baigne dans un paysage de bord de mer. Cette dernière est présente dès les premières lignes du roman : «Zut! s'écria tout à coup le père Roland qui depuis un quart d'heure demeurait immobile, les yeux fixés sur l'eau, et soulevant par moments, d'un mouvement très léger, sa ligne descendue au fond de la mer. » (1999 : 1736 d)

En effet, dans Pierre et Jean le thème de la mer est de la plus haute importance. Ce roman commence par l'arrivée d'un paquebot dans le port du Havre et finit par le départ de Pierre sur La Lorraine : « sa femme se retourna encore une fois pour jeter un dernier regard sur la haute mer ; mais elle ne vit plus rien qu'une petite fumée grise, si lointaine, si légère qu'elle avait l'air d'un peu de brume » (1999 : 2018 c).

Pierre et Jean raconte l'histoire d'une famille qui vit en paix jusqu'au jour où l'on apprend la mort d'un vieil ami. Ce dernier a légué toute sa fortune à Jean, le fils cadet des Roland. C'est là où commence la crise intérieure de son frère Pierre, rongé par la jalousie et le doute.

Dès que l'héritage est annoncé, et que Pierre est obsédé par la jalousie, c'est vers la mer qu'il se dirige : « Puis sur l'eau profonde, sur l'eau sans limites, plus sombre que le ciel, on croyait voir, ça et là, des étoiles. » (1999 : 1785 b)

Pour parler de la mer, Maupassant utilise un vocabulaire précis et même technique. On dirait une leçon de vocabulaire marin :

Et les barques de pêche et les grands voiliers aux matures légères glissant sur le ciel, traînés par d'imperceptibles remorqueurs, arrivaient tous, vite ou lentement, vers cet ogre dévorant, qui, de temps en temps, semblait repu, et rejetait vers la pleine mer une autre flotte de paquebots, de briques, de goélettes, de trois-mâts chargés de ramures emmêlées. Les steamers hâtifs s'enfuyaient à droite, à gauche, sur le ventre plat de l'océan, tandis que les bâtiments à voiles, abandonnés par les mouches qui les avaient halés, demeuraient immobiles, tout en s’habillant de la grande hune au petit perroquet, de toile blanche ou de toile brune qui semblait rouge au soleil couchant. (1999 : 1757 a).

C'est ainsi, qu'en une seule page, Maupassant évoque les barques de pêche et les grands voiliers. Il distingue parmi les bateaux, des remorqueurs, des paquebots, des briques, des goélettes, des trois-mâts et des steamers. Maupassant ne recule pas devant les détails techniques. Il donne des précisions sur le mât appelé « grande hune » et mentionne plusieurs voiles, à titre d'exemple : « le petit perroquet ».

Ainsi, le romancier aime l'exactitude. En diversifiant ce vocabulaire, Maupassant veut que le lecteur voie la variété des bateaux qui peuplent la mer. Il use ainsi d'un lexique spécifique aux hommes de mer par souci du détail. Il veille à ce que le lecteur saisisse toutes les nuances qui rendent le cadre réaliste.

Comme cela a déjà été souligné dans l'analyse du vocabulaire spécifique de Mont-Oriol, l'eau a fréquemment une fonction symbolique. C'est encore le cas dans Pierre et Jean : Pierre confond le plus souvent les deux termes mer et mère.

Pierre contemple la rade de nuit, rencontre Jean sur la jetée. Il part furieux. La présence de son frère le prive de sa mère : "Lorsqu' il fut rentré dans la ville, il se demanda de nouveau ce qu’il ferait, mécontent de cette promenade écourtée, d’avoir été privé de la mer par la présence de son frère. » (1999 : 1789 a)

Dans le chapitre $\mathrm{V}$, alors que la famille était en excursion à Trouville, Pierre déduit que sa mère avait caché le portrait du Maréchal lorsque la ressemblance de Jean avec ce dernier était devenue évidente : « Le mouvement du bateau qui partait troubla sa pensée et la dispersa. Alors, s’étant levé, il regarda la mer. » (1999 : 1878 c).

Dans le passage « la mer invisible ensevelie sous les brouillards » (1999 : 1857 b), c'est la mère qui aux yeux de Pierre n'est plus claire. L'image de la mer qui symbolise la mère est claire dans l'extrait suivant : «Et la Seine descendant de 
Rouen semblait un large bras de mer séparant deux terres voisines. » (1999 : 1879 a). Le bras de mer qui sépare deux terres peut être interprété comme image de la mère qui sépare les deux fils.

Dans Pierre et Jean, l'eau est liée également à la noyade : « Pierre marchait au milieu de ces gens, plus perdu, plus séparé d'eux, plus isolé, plus noyé dans sa pensée torturante, que si on l'avait jeté à la mer du pont d'un navire, à cent lieues au large. » (1999: 1880 a.).

L'eau signifie aussi l'affirmation quand on est le conducteur de son embarcation : "Pendant trois heures, Pierre, tranquille, calme et content, vagabonda sur l'eau frémissante, gouvernant, comme une bête ailée, rapide et docile, cette chose de bois et de toile qui allait et venait à son caprice, sous une pression de ses doigts. » (1999 : 1837 b.).

Ainsi, l'eau est assimilée ici à l'air. Pierre, délivré de ses obsessions, est apaisé de son trouble intérieur sous l'effet de l'eau.

A la fin du roman, Pierre s'abandonne à l'eau en voyageant dans la Lorraine. Il a donc choisi de fuir la vie et d'accepter la mort, car lui seul connait la vérité. La mer est ainsi associée dans Pierre et Jean à des envies de départ. Il doit partir en sachant que son départ est une mort bien décidée.

La mer bleue dans Pierre et Jean peut porter la joie au personnage comme elle peut le conduire à la misère. Cette mer qui représentait au début du roman un espace accueillant et réconfortant devient à la fin de l'œuvre un lieu d'exil et d'isolement. La mer est ainsi un élément de vie et de mort.

L'eau a donc une connotation symbolique dans les deux romans de Maupassant. Dans Mont-Oriol, elle procure le plaisir et la jouissance à Andermatt, alors que dans Pierre et Jean, la mer n'est pas un simple décor dans le roman, elle a une représentation vivante dans toute l'œuvre. Du début du roman à la fin, la mer est liée aux moments de réflexion de Pierre. Mais elle devient à la fin son lieu d'enfermement et d'exil.

\section{Références bibliographiques}

Besnard-Coursodon, Micheline (1973). Étude thématique et structurale de l'œuvre de Maupassant : le piège. Paris : Nizet.

BonNEFIS, Philippe (1981). Comme Maupassant. Lille : Presses Universitaires de Lille.

Brunet, Étienne (1988). Le Vocabulaire de Victor Hugo, Vol .I. Paris - Genève : Champion - Slatkine.

BRUNET, Étienne (2009). À la recherche des Illusions perdues. Compte d'auteurs. Paris : Honoré Champion.

FonYI Antonia (1993). Maupassant. Paris : Kimé.

GiacChetTI, Claudine (1993). Maupassant, espaces du roman. Paris : Droz.

HeLms, Laure (2008). Pierre et Jean de Guy de Maupassant. Paris : Gallimard.

KAstBerg-SJOBlom, Margareta (2006). L'écriture de J.M.G. Le Clézio : Des mots aux thèmes. Paris : Honoré de Champion.

MagRi, Véronique. (1995). Le Discours sur l'autre. Paris : Honoré de Champion.

Maupassant, Guy (1977). Une vie. Paris : Presses-Pocket.

Maupassant, Guy (1983). Fort comme la mort. Paris : Albin Michel.

MAupassant, Guy (1993). Notre cour. Paris : Gallimard.

Maupassant, Guy (1999). Pierre et Jean. Paris : Albin Michel.

Maupassant, Guy (2002). Mont-Oriol. Paris : Gallimard.

Maupassant, Guy (2006). Bel-Ami. Paris : Pocket.

Maupassant, Guy (2006). Carnets de Voyage : Au soleil, Sur l'eau, La vie errante. Paris : Editions Rive Droite.

MAYNiAL, Édouard (1935). La vie et l'œuvre de Guy de Maupassant. Paris : Mercure de France.

MAYnial, Édouard (1935). La vie et l'œuvre de Guy de Maupassant. Paris : Mercure de France.

Michel, Mougenot (1992). Pierre et Jean, Guy de Maupassant. Paris : Parcours de lecture.

QUESNEL, Alain (1999). Les Romans de Maupassant. Paris : PUF. 